\title{
Rates of infection after colonoscopy and osophagogastroduodenoscopy in ambulatory surgery centres in the USA
}

\author{
Peiqi Wang, ${ }^{1,2} \operatorname{Tim~Xu}^{3}{ }^{3}$ Saowanee Ngamruengphong, ${ }^{1}$ Martin A Makary, ${ }^{4,5}$ \\ Anthony Kalloo, ${ }^{1}$ Susan Hutfless ${ }^{1,2,6}$
}

- Additional material is published online only. To view please visit the journal online (http://dx.doi.org/10.1136/ gutjnl-2017-315308).

'Department of Medicine, Division of Gastroenterology and Hepatology, Johns Hopkins University, Baltimore, Maryland, USA

${ }^{2}$ Gastrointestinal Epidemiology Research Center, Johns Hopkins University, Baltimore, Maryland, USA

${ }^{3}$ McKinsey \& Company, Washington, District of Columbia, USA

${ }^{4}$ Department of Surgery, Johns Hopkins Hospital, Baltimore, Maryland, USA

${ }^{5}$ Department of Health Policy Management, Johns Hopkins School of Public Health, Baltimore, Maryland, USA ${ }^{6}$ Department of Epidemiology, Johns Hopkins University Bloomberg School of Public Health, Baltimore, Maryland, USA

\section{Correspondence to} Dr Susan Hutfless, Division of Gastroenterology and Hepatology, Johns Hopkins University, Baltimore, MD 21287, USA; shutfle1@jhmi.edu

Received 19 September 2017 Revised 18 April 2018 Accepted 3 May 2018 Published Online First 18 May 2018

Check for updates

To cite: Wang $P, X u T$, Ngamruengphong $S$, et al. Gut 2018;67:1626-1636.

\section{ABSTRACT \\ Objective Over 15 million colonoscopies and 7 million} osophagogastroduodenoscopies (OGDs) are performed annually in the USA. We aimed to estimate the rates of infections after colonoscopy and OGD performed in ambulatory surgery centres (ASCs).

Design We identified colonoscopy and OGD procedures performed at ASCs in 2014 all-payer claims data from six states in the USA. Screening mammography, prostate cancer screening, bronchoscopy and cystoscopy procedures were comparators. We tracked infectionrelated emergency department visits and unplanned in-patient admissions within 7 and 30 days after the procedures, examined infection sites and organisms and analysed predictors of infections. We investigated casemix adjusted variation in infection rates by ASC.

Results The rates of postendoscopic infection per 1000 procedures within 7 days were 1.1 for screening colonoscopy, 1.6 for non-screening colonoscopy and 3.0 for OGD; all higher than screening mammography (0.6) but lower than bronchoscopy (15.6) and cystoscopy (4.4) $(p<0.0001)$. Predictors of postendoscopic infection included recent history of hospitalisation or endoscopic procedure; concurrence with another endoscopic procedure; low procedure volume or non-freestanding ASC; younger or older age; black or Native American race and male sex. Rates of 7-day postendoscopic infections varied widely by ASC, ranging from 0 to 115 per 1000 procedures for screening colonoscopy, 0 to 132 for nonscreening colonoscopy and 0 to 62 for OGD.

Conclusion We found that postendoscopic infections are more common than previously thought and vary widely by facility. Although screening colonoscopy is not without risk, the risk is lower than diagnostic endoscopic procedures.

\section{INTRODUCTION}

On 19 February 2015, the Food \& Drug Administration (FDA) published a Safety Communication relating to possible transmission of microorganisms from reprocessed duodenoscopes used for endoscopic retrograde cholangiopancreatography (ERCP). ${ }^{1}$ Between January 2013 and December 2014, the FDA received 75 Medical Device Reports encompassing infections of approximately 135 patients in the USA. These infectious agents included multidrug-resistant bacteria such as Escherichia coli and Klebsiella species. The infections were originally thought to be due to design flaws, but an outbreak occurred in a facility that used the redesigned duodenoscope and revised cleaning protocol. $^{2}$ Other mechanisms might play a role to contribute to postendoscopic infections. ${ }^{3}$ If facility factors play a role in infection transmission, we expect that procedures using colonoscopes and upper endoscopes without elevator mechanisms might also be associated with postendoscopic infections.

While about 500000 ERCP procedures are performed in the USA each year, there are over 15 million colonoscopies and 7 million osophagogastroduodenoscopies (OGDs) performed annually. ${ }^{145}$ About 40\% (6.3 million) of colonoscopies are performed for colorectal cancer screening among individuals who are completely asymptomatic. Rates of colonoscopy-associated complications such as bleeding and perforation have been studied. ${ }^{6-9}$ A meta-analysis of 21 studies published between 2001 and 2015 estimated incidences for postcolonoscopy perforation, bleeding and mortality to be $0.5 / 1000,2.6 / 1000$ and $2.9 / 100000$ procedures. $^{10}$ Case reports have described infections after colonoscopy and OGD performed in hospitals, which tend to have sicker patients than ambulatory surgery centres (ASCs) and infection control units monitoring for outbreaks. No study has examined the rate of infections after colonoscopy or OGD. In particular, no study or case report has examined rates of infections after screening colonoscopy, non-screening colonoscopy or OGD performed in ASCs. Over $50 \%$ of endoscopic procedures are now performed in ASCs in the USA. ${ }^{11}$ Healthcare reform has led to a shift in endoscopic procedures from hospitals to ASCs to reduce costs of care. ${ }^{12}$

We aimed to examine the variation in rates of infections after screening and non-screening colonoscopy and OGD performed in ASCs in 2014. We used all-payer information from six geographically and racially diverse states representing $31 \%$ of the US population. We also examined the infections by site and organism and analysed patient, procedure and facility predictors of infection.

\section{METHODS}

\section{Data sources and linkage}

We identified colonoscopy and OGD procedures performed in ASCs and tracked patients' emergency 
department (ED) visits and hospitalisations within 7 and 30 days after the endoscopic procedures. We obtained statewide ASC, ED and in-patient claims data from six states that varied in population size and race. For Florida, New York, Georgia, Nebraska and Vermont, we used Agency for Healthcare Research and Quality (AHRQ) Healthcare Cost and Utilization Project (HCUP) data. For California, we used data collected by the Office of Statewide Health Planning and Development (OSHPD). Information from both hospital-owned and freestanding ASCs was available in California, Florida and New York. Only hospital-owned ASC information was available from Nebraska, Vermont and Georgia. The variables in HCUP and OSHPD data were sufficiently uniform to be merged for analysis, including variables on patient characteristics and service utilisation information. ED visits and hospitalisations were linked to eligible endoscopic procedures using unique encrypted patient identifiers and deidentified service utilisation dates.

\section{Endoscopic procedures}

We included colonoscopy and OGD procedures based on Current Procedural Terminology (CPT) codes (online supplementary appendix tables 1 and 2). We excluded procedures where information was missing for age $(n=10)$, sex $(n=79)$, patient identifier $(n=135997)$ or procedure date $(n=28)$ and where an infection diagnosis code was present at the time of the procedure (online supplementary appendix table $3 ; n=50$ 928) and during which the patient died $(\mathrm{n}=13)$. Screening colonoscopy was identified using CPT codes G0105 and G0121. For New York, Nebraska and Vermont where CPT modifiers were reported, if the modifier ' 33 ' or 'PT' was appended to any colonoscopy CPT code (online supplementary appendix tables $1),{ }^{13}$ we classified this procedure as screening colonoscopy. These modifiers identify colorectal cancer screening tests that were converted to diagnostic or therapeutic procedures. For California, Florida and Georgia where no CPT modifiers were reported, the CPT code 45378 was considered a screening colonoscopy in addition to G0105 and G0121.

\section{Comparator groups to calculate the background infection rates and comparison to other endoscopic procedures}

Considering that the population is at risk for infection-related visits even without undergoing a colonoscopy or OGD, we selected patients undergoing screening mammography and prostate cancer screening (CPT codes given in online supplementary appendix table 3) as non-endoscopic comparator groups that do not involve sedation. Screening mammography was selected as the primary comparator as most prostate cancer screenings do not occur in ASCs. As a non-invasive examination often performed on a healthy population, these screening tests provided the opportunity to estimate the background rate of infection that would occur stochastically after a visit at an ASC, which was helpful for understanding the net influence of colonoscopy and OGD on infection outcomes. We then compared the rates with bronchoscopy and cystoscopy (online supplementary appendix table 3). These procedures are common endoscopic procedures that involve sedation and often occur in the same ASC as gastrointestinal endoscopic procedures.

\section{Unplanned visits}

All-cause unplanned visits within 7 and 30 days after procedures were defined according to the Centers of Medicare and Medicaid Services (CMS) colonoscopy quality measure (ID 2086: Facility 7 Day Risk-Standardized Hospital Visit Rate after Outpatient
Significance of this study

What is already known on this subject?

- Previous studies estimated rates of colonoscopy-associated complications such as bleeding, perforation and aspiration pneumonia, but not infectious complications.

- Case reports have described infection outbreaks after colonoscopy and osophagogastroduodenoscopy (OGD) performed in hospitals.

- No study has comprehensively examined the rates of infections after colonoscopy or OGD in ambulatory surgery centres (ASCs). Unlike hospitals, ASCs often do not have infection control units and do not have linked electronic medical records to local emergency departments and hospitals to identify postprocedural events.

What are the new findings?

- This study aimed to estimate the rates of infection after colonoscopy and OGD and compare those rates with screening mammography, prostate cancer screening, bronchoscopy and cystoscopy.

- Although patients are routinely told that common endoscopic procedures are entirely safe, we found that postendoscopic infections (those present within 7 or 30 days after the procedure) are more common than previously thought and vary widely by the ASC facility.

- The observed postendoscopic infection rates at some ASCs are over 100 times higher than their expected rates that account for patient severity and procedure complexity.

- Overall and site-specific infection rates are 2-10 times higher for colonoscopy and OGD compared with screening mammography.

- Infection rates after colonoscopy and OGD were lower compared with bronchoscopy and cystoscopy.

How might it impact on clinical practice in the foreseeable future?

- Patients should be informed of the infectious risk associated with screening colonoscopy and all endoscopic procedures.

- This study provides comparative rates to common nonendoscopic and endoscopic procedures to inform those patient-provider conversations.

- This study also highlights that the facility that a patient undergoes their procedure impacts their risk of infection.

- Patient-accessible public reporting of facility-level procedure volumes and infection rates may be valuable to patients seeking quality of care information.

Colonoscopy) (online supplementary appendix figure 1). ${ }^{14}{ }^{15}$ All ED visits and the hospitalisations with evidence of observational stay service utilisation were considered unplanned visits. For hospitalisations without an observational stay, CMS's Planned Readmission Algorithm, V.4.0 was used to remove the planned admissions. ${ }^{15}$

\section{Infections}

Our analyses focused on unplanned visits associated with infections (online supplementary appendix table 4). Our primary outcome was 7-day infection-related unplanned visit rates. Thirty-day rates were also calculated. We examined infections by the sites involved, including gastrointestinal, respiratory, genitourinary and central nervous systems as well as septicaemia and infectious endocarditis. We also investigated infections by the 
responsible organism, namely drug-resistant microorganisms such as E. coli, Klebsiella pneumoniae, Clostridium difficile, Pseudomonas, Staphylococci, Streptococci, gram-negative and anaerobic bacteria.

\section{Aspiration pneumonia}

We investigated unplanned visits related to aspiration pneumonia (online supplementary appendix table 5), because it is a potential complication due to the anaesthesia or sedation used for colonoscopy and OGD and could be miscoded as infectious pneumonia. The definition of aspiration pneumonia using The International Classification of Diseases, Ninth Revision, Clinical Modification (ICD-9-CM) codes included two possible case definitions in previous publications. ${ }^{16-18}$ For completeness, we examined all ICD-9-CM codes that could potentially be coded for aspiration pneumonia and calculated the rates for two case definitions: (1) 507.0 or (2) 507.0, 507.8, 482.89, 482.9 or 483.8. The case definition of 507.0 for aspiration pneumonia was confirmed in $91 \%$ of patients during chart review. ${ }^{18}$ For the state of New York where method of anaesthesia was reported, we compared the rates of infectious and aspiration pneumonia between procedures performed under general anaesthesia versus those not associated with general anaesthesia.

\section{Patient predictors}

We examined patient, procedure and facility predictors of postendoscopic infection-related unplanned visits. Patient characteristics that may impact the risk of infections included age; sex; race; history of hospitalisation and gastrointestinal endoscopic procedure within 30 days prior to procedure; conditions present at the procedural visit that were identified by the Elixhauser comorbidity index ${ }^{19}$ and inflammatory bowel disease (ICD-9-CM codes 555 and 556). Elixhauser comorbidities were categorised by the number of patient conditions $(0,1$ or $2-14$ (the maximum number observed)). We imputed the race of all patients in Nebraska to white as $96 \%$ of the population is white and no race information was available.

\section{Procedure predictors}

Procedure characteristics included invasiveness and concurrence with other gastrointestinal endoscopic procedures on the same day. We determined a procedure's invasiveness according to whether gastrointestinal mucosa was likely disrupted during the procedure (online supplementary appendix tables 1 and 2). For example, screening and diagnostic procedures where no biopsy was taken were categorised as non-invasive. However, diagnostic procedures where biopsies were taken or therapeutic procedures like polypectomy and lesion ablation occurred were categorised as invasive. Concurrence with other gastrointestinal endoscopic procedures was determined by examining whether another endoscopic procedure was coded on the same visit. For example, if a colonoscopy and an OGD occurred on the same visit, then both procedures contributed to the analyses and were considered as having concurred with another gastrointestinal endoscopic procedure.

\section{Facility predictors}

Facility characteristics of interest included the state where the ASC was located; annual procedure volume; hospital-owned or freestanding; multispecialty or gastroenterology-specific endoscopy unit and the proportion of gastrointestinal endoscopic procedures that were performed on patients with an infection coded on the day of the procedure. We calculated the 2014 colonoscopy and OGD volumes for each ASC and categorised the volumes into tertiles. We examined whether non-gastrointestinal endoscopic procedures (bronchoscopy and cystoscopy) were performed at each ASC and defined facilities that performed both gastrointestinal and non-gastrointestinal endoscopic procedures as multispecialty. Multispecialty facilities are likely to share procedural suites and cleaning rooms for all scopes, which could lead to cross-contamination. Although procedures with infection diagnoses coded on the day of the procedure were excluded from the analyses, we calculated the annual cumulative proportion of procedures performed on infectious cases for each ASC as a predictor.

\section{Statistical analyses}

\section{Six-state outcome rates}

We calculated the unadjusted rates of 7-day postendoscopic infection-related unplanned visits for screening and non-screening colonoscopy, OGD, screening mammography, bronchoscopy and cystoscopy. Thirty-day infection and all-cause unplanned visit rates were calculated for screening and non-screening colonoscopy, OGD and the primary comparator, screening mammography. The denominator was the eligible screening or non-screening colonoscopy; OGD procedures or comparator procedures in all six states. The numerator was the number of procedures that were followed by an ED visit or unplanned hospitalisation where at least one infection was coded within 7 or 30 days. Because planned hospitalisations were not considered outcome events, procedures followed by planned hospitalisations that included an infection code were not included in the numerator but they were part of the denominator. The proportion of infection-related unplanned visits that resulted in hospitalisation and the associated length of stay and mortality were calculated for the gastrointestinal endoscopic procedures.

\section{Patient, procedure and facility predictors of infection-related} unplanned visits

To analyse the predictors of 7-day infection-related unplanned visits, we used multivariable logistic regression models with patient, procedure and facility characteristics as predictors. We also used the same models to analyse the predictors of 7-day unplanned visits specifically related to infections.

\section{Variation in infection-related unplanned visit rates by ASC}

We used multivariable logistic regression models with patient and procedure predictors to compare the observed versus expected number of events by centre. We summed the modelbased expected probabilities of infection-related unplanned visits for all procedures performed at each ASC to get the expected number of events. We then calculated the ratio of the observed number of events over the expected for each ASC. Adjusted outcome rates were calculated by multiplying the observed over expected ratio by the six-state unadjusted outcome rates. We plotted the ASC variation in 7-day infection rates after screening colonoscopy, non-screening colonoscopy and OGD procedures for ASCs that performed at least 10 of the procedures in 2014. We conducted statistical analyses using SAS software, V.9.4 (Cary, North Carolina, USA) and Stata V.13 (College Station, Texas, USA).

\section{RESULTS}

There were 462068 screening colonoscopies performed at 1157 ASCs and 914140 non-screening colonoscopies performed at 1202 ASCs (table 1). There were 873138 OGDs performed 
Table 1 Infection-related and all-cause unplanned visit rates after colonoscopy, OGD and the comparator tests and procedures

\begin{tabular}{|c|c|c|c|c|c|c|c|}
\hline Rate per 1000 procedures & $\begin{array}{l}\text { Screening } \\
\text { colonoscopy } \\
(n=462068)\end{array}$ & $\begin{array}{l}\text { Non-screening } \\
\text { colonoscopy } \\
(n=914140)\end{array}$ & $\begin{array}{l}\text { OGD } \\
(n=873138)\end{array}$ & $\begin{array}{l}\text { Screening } \\
\text { mammography } \\
(n=647212)\end{array}$ & $\begin{array}{l}\text { Prostate cancer } \\
\text { screening } \\
(n=26428)\end{array}$ & $\begin{array}{l}\text { Bronchoscopy } \\
(n=30116)\end{array}$ & $\begin{array}{l}\text { Cystoscopy } \\
(n=68432)\end{array}$ \\
\hline All-cause visits & 11.613 & 16.414 & 34.606 & 6.435 & 13.735 & 51.036 & 37.906 \\
\hline Infection-related visits & 1.128 & 1.566 & 3.038 & 0.609 & 1.551 & 16.536 & 4.413 \\
\hline Infections of gastrointestinal system & 0.160 & 0.290 & 0.354 & 0.039 & 0.076 & 0.299 & 0.321 \\
\hline Infections of genitourinary system & 0.045 & 0.048 & 0.087 & 0.025 & 0.038 & 0.033 & 0.789 \\
\hline Infections of respiratory system & 0.524 & 0.602 & 1.260 & 0.351 & 0.908 & 12.385 & 0.950 \\
\hline Pneumonia & 0.299 & 0.286 & 0.688 & 0.104 & 0.341 & 11.223 & 0.555 \\
\hline Aspiration pneumonia & 0.087 & 0.097 & 0.333 & 0.015 & 0 & 1.527 & 0.088 \\
\hline Infections outside the treated organ system & $0.968^{*}$ & $1.276^{*}$ & $2.684^{*}$ & $0.609 \dagger$ & $1.551 \dagger$ & $4.151 \neq$ & $3.624 \S$ \\
\hline
\end{tabular}

*Non-gastrointestinal infections for colonoscopy and OGD (all infections minus gastrointestinal infections).

†All infections for screening mammography.

¥Non-respiratory infections for bronchoscopy (all infections minus respiratory infections).

$\S$ Non-genitourinary infections for cystoscopy (all infections minus genitourinary infections).

OGD, osophagogastroduodenoscopy.

at 1212 ASCs. The comparator groups consisted of 647212 screening mammographies at 338 ASCs; 26428 prostate cancer screenings at 209 ASCs; 30116 bronchoscopies at 665 ASCs and 68432 cystoscopies at 912 ASCs. The number of ASCs, maximum procedure volume and patient characteristics such as race varied by state as was expected given the diversity of the states selected (online supplementary appendix table 6).

\section{Infection-related unplanned visits rates}

The rates of 7-day infection-related unplanned visits were 1.1 per 1000 procedures for screening colonoscopy, 1.6 for non-screening colonoscopy and 3.0 for OGD, which were twofold to fivefold higher compared with the rate of 0.6 for screening mammography and similar to twofold higher compared with the rate of 1.6 for prostate screening (table 1 ). The rates of 30-day infection-related unplanned visits per 1000 procedures were 4.0 for screening colonoscopy, 5.4 for non-screening colonoscopy and 10.8 for OGD, compared with 2.9 for screening mammography (table 2). The proportion of infection-related unplanned visits that resulted in hospitalisation was $61.8 \%$ for screening, $60.5 \%$ for non-screening colonoscopy and $64.2 \%$ for OGD. Infection-related hospitalisations required a mean length of stay of 8 days (range: 0-54, median 5) for screening colonoscopy, 7 days (range: 0-98, median 5) for non-screening colonoscopy and 8 days for OGD procedures (range: 0-148, median 5). Death occurred during $0.4 \%, 1.7 \%$ and $2.6 \%$ of the infection-related unplanned visits after screening colonoscopy, non-screening colonoscopy and OGD procedures.

\section{Infections by site and by organism}

The rates of gastrointestinal infections were 0.2 per 1000 procedures for screening colonoscopy, 0.3 for non-screening colonoscopy and 0.4 for OGD, 5-10 times higher than the rate of 0.04 per 1000 procedures for screening mammography (table 2). The rates of septicaemia and infections of respiratory and genitourinary systems were also higher for screening and non-screening colonoscopy and OGD compared with screening mammography. E. coli, C. difficile and Staphylococci were the most common organisms for screening and non-screening colonoscopy and OGD, with infection rates 3-30 times higher compared with screening mammography. The most common infections within 30 days after procedures were similar to those within 7 days.
We examined the rates of acute and subacute bacterial or infective endocarditis for comparison with the prior case reports (table 2). We identified 7 and 21 unplanned visits for acute and subacute bacterial or infective endocarditis within 7 and 30 days after non-screening colonoscopy (7 day rate: 0.008 per 1000 procedures; 30 day rate: 0.023$)$ and 6 and 38 after OGD (7 day rate: 0.007 ; 30 day rate: 0.044$)$. There was no visit for bacterial/infective endocarditis after screening colonoscopy within 7 days and 5 visits within 30 days (30 day rate: 0.011). There was no visit for bacterial/infective endocarditis after screening mammography within 7 or 30 days.

\section{Aspiration pneumonia after gastrointestinal endoscopic procedures, bronchoscopy and cystoscopy}

The aspiration pneumonia rate was lowest after screening colonoscopy and highest after bronchoscopy (screening colonoscopy 0.087; cystoscopy 0.088 ; non-screening colonoscopy 0.097 ; OGD 0.333 and bronchoscopy 1.527) (table 1). When infections of the treated organ system (ie, infections that could possibly be indications) were excluded, the infection rates were still lower for colonoscopy and OGD than bronchoscopy and cystoscopy. The majority of the codes for aspiration pneumonia were 507.0 (online supplementary appendix table 7).

\section{Influence of general anaesthesia}

New York was the only state that reported the anaesthesia method used for endoscopic procedures (online supplementary appendix table 8 ). The rates of infections after procedures performed under general anaesthesia were largely similar to those not performed under general anaesthesia. The rates of respiratory system infection per 1000 procedures with versus without general anaesthesia were 0.377 vs 0.347 for screening colonoscopy $(\mathrm{p}=0.86), 0.405$ vs 0.516 for non-screening colonoscopy $(p=0.34)$ and 1.071 vs 0.790 for OGD $(p=0.07)$. For colonoscopy, the rates of aspiration pneumonia were similar regardless of the use of general anaesthesia (rates per 1000 procedures and $\mathrm{p}$ values (general anaesthesia vs not): screening colonoscopy: 0 vs $0.053, p=0.40$; non-screening colonoscopy: 0.119 vs 0.058 , $\mathrm{p}=0.15)$. For OGD, the rates of aspiration pneumonia was about twofold higher with the use of general anaesthesia (rates per 1000 procedures and $\mathrm{p}$ value (general anaesthesia vs not): 0.341 vs $0.179, \mathrm{p}=0.03$ ). 
Table 2 Seven-day infection-related unplanned visit rates (by major infection sites and organisms) after colonoscopy, OGD or screening mammography at an ambulatory surgery centre, 2014

\begin{tabular}{|c|c|c|c|c|c|c|c|c|}
\hline \multirow[b]{2}{*}{ Infection (ICD-9-CM codes) } & \multicolumn{2}{|c|}{$\begin{array}{l}\text { Screening colonoscopy } \\
(\mathrm{n}=462068)\end{array}$} & \multicolumn{2}{|c|}{$\begin{array}{l}\text { Non-screening colonoscopy } \\
(\mathrm{n}=914140)\end{array}$} & \multicolumn{2}{|c|}{$\begin{array}{l}\text { OGD } \\
(n=873138)\end{array}$} & \multicolumn{2}{|c|}{$\begin{array}{l}\text { Screening } \\
\text { mammography } \\
(\mathrm{n}=647212)\end{array}$} \\
\hline & $\mathrm{N}$ of visits & $\begin{array}{l}\text { Rate/1000 } \\
\text { procedures }\end{array}$ & $\mathrm{N}$ of visits & $\begin{array}{l}\text { Rate/1000 } \\
\text { procedures }\end{array}$ & $\mathrm{N}$ of visits & $\begin{array}{l}\text { Rate/1000 } \\
\text { procedures }\end{array}$ & $\mathrm{N}$ of visits & $\begin{array}{l}\text { Rate/1000 } \\
\text { procedures }\end{array}$ \\
\hline \multicolumn{9}{|l|}{ By infection site } \\
\hline Infections of gastrointestinal system (001-009, & 74 & & 265 & & 309 & & 25 & \\
\hline $566-567)$ & & 0.160 & & 0.290 & & 0.354 & & 0.039 \\
\hline Intestinal infectious diseases (001-009) & 24 & 0.052 & 130 & 0.142 & 182 & 0.208 & 20 & 0.031 \\
\hline Abscess of anal and rectal regions (566) & 4 & 0.009 & 13 & 0.014 & 5 & 0.006 & 3 & 0.005 \\
\hline Peritonitis and retroperitoneal infections (567) & 47 & 0.102 & 125 & 0.137 & 126 & 0.144 & 2 & 0.003 \\
\hline $\begin{array}{l}\text { Infections of respiratory system ( } 460-466,480-488 \text {, } \\
511.1)\end{array}$ & 242 & 0.524 & 550 & 0.602 & 1100 & 1.260 & 227 & 0.351 \\
\hline Acute respiratory infections (460-466) & 97 & 0.210 & 265 & 0.290 & 482 & 0.552 & 152 & 0.235 \\
\hline Pneumonia (480-486) & 138 & 0.299 & 261 & 0.286 & 601 & 0.688 & 67 & 0.104 \\
\hline Influenza (487-488) & 14 & 0.030 & 34 & 0.037 & 43 & 0.049 & 13 & 0.020 \\
\hline $\begin{array}{l}\text { Pleurisy with effusion, with mention of a bacterial } \\
\text { cause other than tuberculosis (511.1) }\end{array}$ & 0 & 0 & 0 & 0 & 1 & 0.001 & 0 & 0 \\
\hline Septicaemia (038) & 88 & 0.190 & 235 & 0.257 & 568 & 0.651 & 25 & 0.039 \\
\hline Infections of genitourinary system (590) & 21 & 0.045 & 44 & 0.048 & 76 & 0.087 & 16 & 0.025 \\
\hline Bacterial/infective endocarditis $(421.0,421.1)$ & 0 & 0 & 7 & 0.008 & 6 & 0.007 & 0 & 0 \\
\hline $\begin{array}{l}\text { Infections of central nervous system }(320,321,323.0 \text { - } \\
323.2,323.4,323.61)\end{array}$ & 0 & 0 & 2 & 0.002 & 1 & 0.001 & 1 & 0.002 \\
\hline \multicolumn{9}{|l|}{ By organism } \\
\hline Infection with drug-resistant microorganisms (V09) & 1 & 0.002 & 4 & 0.004 & 22 & 0.025 & 0 & 0 \\
\hline Escherichia coli $(038.42,041.4,482.82)$ & 39 & 0.084 & 102 & 0.112 & 174 & 0.199 & 21 & 0.032 \\
\hline Klebsiella pneumoniae $(041.3,482.0)$ & 10 & 0.022 & 20 & 0.022 & 56 & 0.064 & 5 & 0.008 \\
\hline Clostridium difficile (008.45) & 16 & 0.035 & 80 & 0.088 & 113 & 0.129 & 2 & 0.003 \\
\hline Pseudomonas $(038.43,041.7,482.1)$ & 6 & 0.013 & 7 & 0.008 & 41 & 0.047 & 1 & 0.002 \\
\hline Staphylococcus (038.1, 041.1, 482.4) & 22 & 0.048 & 45 & 0.049 & 112 & 0.128 & 9 & 0.014 \\
\hline Streptococcus $(038.0,038.2,041.0,041.2,481,482.3)$ & 12 & 0.026 & 33 & 0.036 & 95 & 0.109 & 4 & 0.006 \\
\hline Gram-negative bacteria $(038.4,482.83)$ & 14 & 0.030 & 41 & 0.045 & 109 & 0.125 & 3 & 0.005 \\
\hline Anaerobes $(038.3,482.81)$ & 0 & 0 & 5 & 0.005 & 5 & 0.006 & 0 & 0 \\
\hline Human papillomavirus (079.4) & 0 & 0 & 3 & 0.003 & 0 & 0 & 0 & 0 \\
\hline
\end{tabular}

ICD-9-CM, The International Classification of Diseases, Ninth Revision, Clinical Modification; OGD, osophagogastroduodenoscopy .

\section{Predictors of 7-day postendoscopic infection-related unplanned visits}

Patient predictors

Age, sex, race, comorbidities and 30-day hospitalisation and endoscopic procedure history had statistically significant associations with 7-day postendoscopic infections (tables 3 and 4). History of hospitalisation within 30 days prior to the procedure was the strongest patient risk factor for postendoscopic infections followed by history of a gastrointestinal endoscopic procedure in the prior 30 days. For screening colonoscopy, patients 70 years and older had higher infection rates compared with those aged 50-59 years old. For non-screening colonoscopy and OGD, both younger and older age groups had higher infection rates than those aged 50-59 years old. Sex did not have any statistically significant associations with postendoscopic infections after screening or non-screening colonoscopy, although female patients were less likely to have an infection after OGD compared with male patients. Black race had higher infection rates compared with the white after screening and non-screening colonoscopy and OGD. Native American had higher rates of infection after screening colonoscopy compared with the white. Number of comorbidities and inflammatory bowel disease were also associated with higher odds of postendoscopic infections.

\section{Procedure predictors}

Invasiveness (eg, polyp removal) did not alter the risk of infection after screening colonoscopy (aOR (95\% CI): 1.16 (0.82 to 1.65$)$ ) or non-screening colonoscopy (aOR (95\% CI): $1.03(0.87$ to 1.22$)$ ), but lowered the risk of infections after OGD (aOR (95\% CI): 0.82 (0.74 to 0.91)) (table 4). Concurrence with another gastrointestinal endoscopic procedure on the same day increased the odds of infections after screening colonoscopy (aOR (95\% CI): 1.40 (1.14 to 1.71)) and non-screening colonoscopy (aOR (95\% CI): 1.62 (1.44 to 1.82$)$ ), but decreased odds of infections after OGD (aOR $(95 \% \mathrm{CI}): 0.78(0.72$ to 0.85$))$.

\section{Facility predictors}

Procedure volume was the strongest predictor of postendoscopic infections (table 4). ASCs with higher volume had the lowest rates of postendoscopic infections. ASCs in New York had lower infection rates compared with those in Florida. ASCs that treated patients with active infection at the time of the procedure did not have statistically significant higher rates of postendoscopic infections.

The patient, procedure and facility predictors of site-specific infections, such as gastrointestinal and respiratory system 
Table 3 Stratified infection-related and all-cause unplanned visit rates after colonoscopy, OGD or screening mammography at an ambulatory surgery centre stratified by prior hospitalisations and procedures, 2014

\section{All eligible patients}

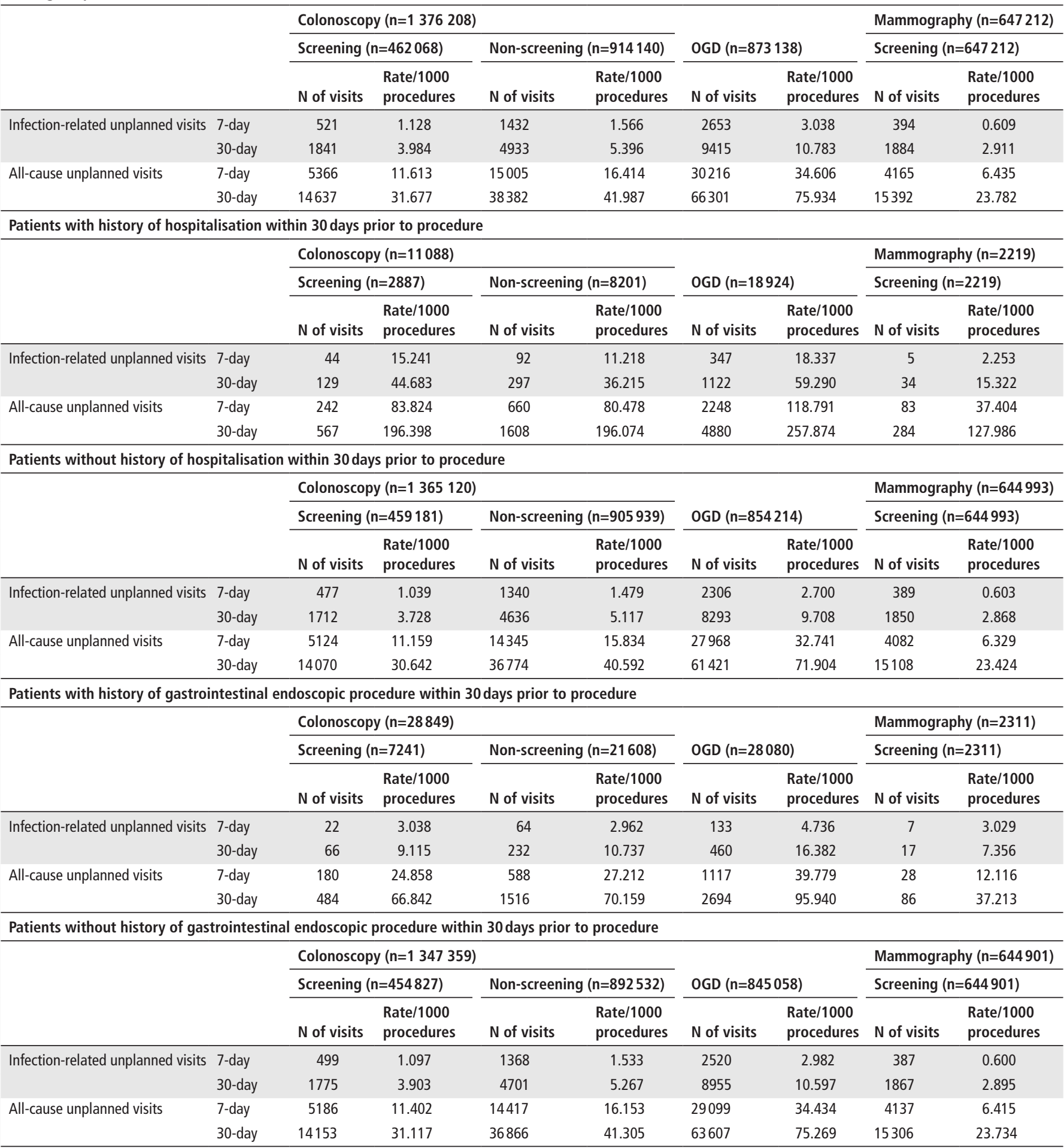

OGD, osophagogastroduodenoscopy.

infections, were similar in direction and magnitude to the all infection results, except with wider CI (data not shown).

Variation in postendoscopic infection-related unplanned visits ASCs with lower procedure volume had higher infection-related unplanned visit rates even after accounting for patient and procedure complexity (figure 1). There was wide variation in the adjusted rates of postendoscopic infections. For the 1047 ASCs that performed 10 or more screening colonoscopies in 2014 , their facility-level case-mix adjusted rates of infection-related unplanned visits ranged from 0 to 115 per 1000 procedures. For the 1140 ASCs that performed 10 or more non-screening colonoscopies, their adjusted infection rates ranged from 0 to 132 per 1000 procedures. Adjusted rates for the 1151 ASCs that 


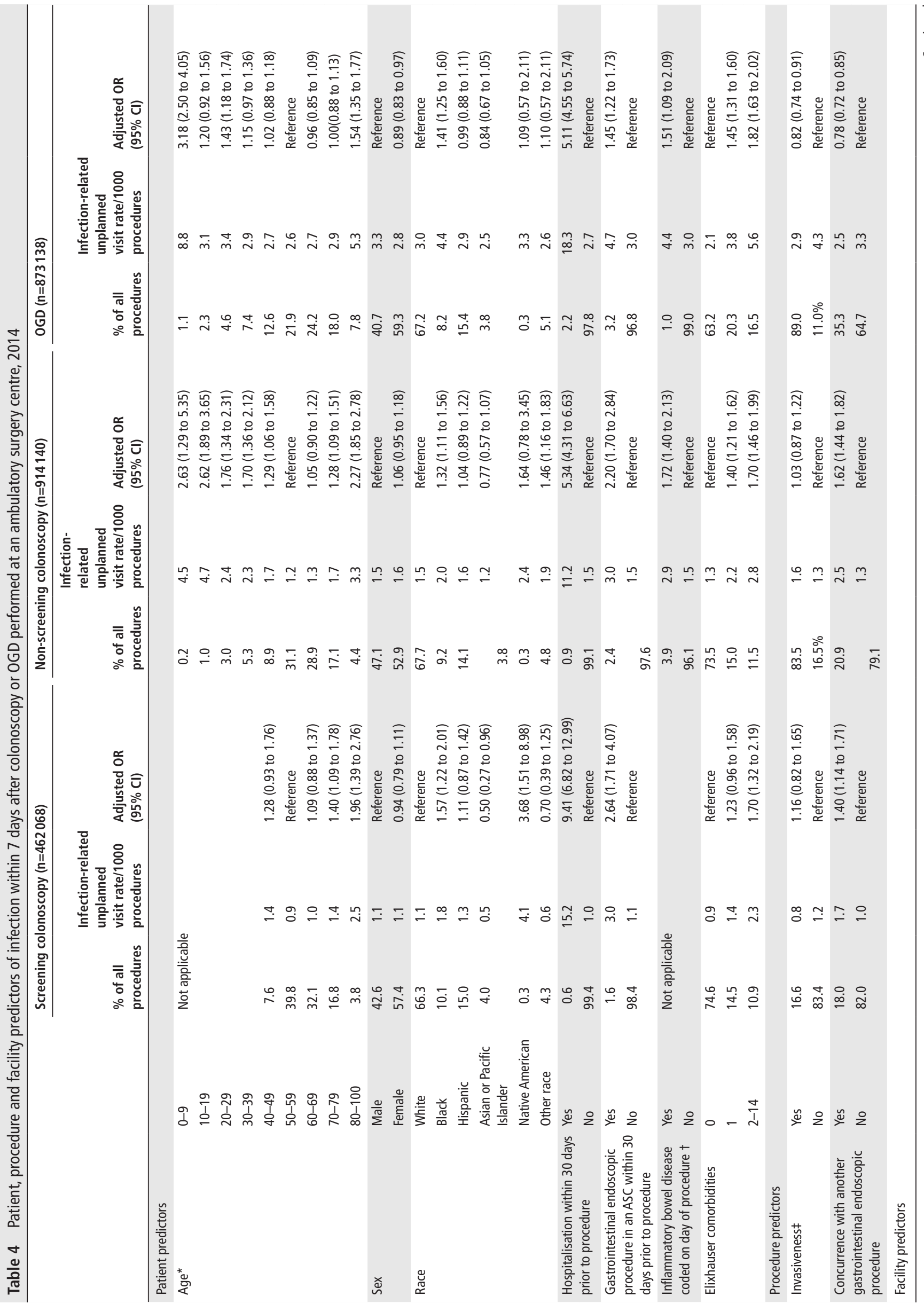

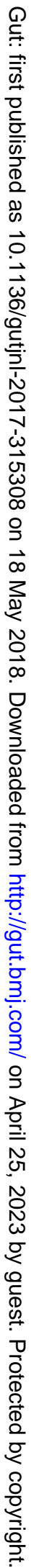




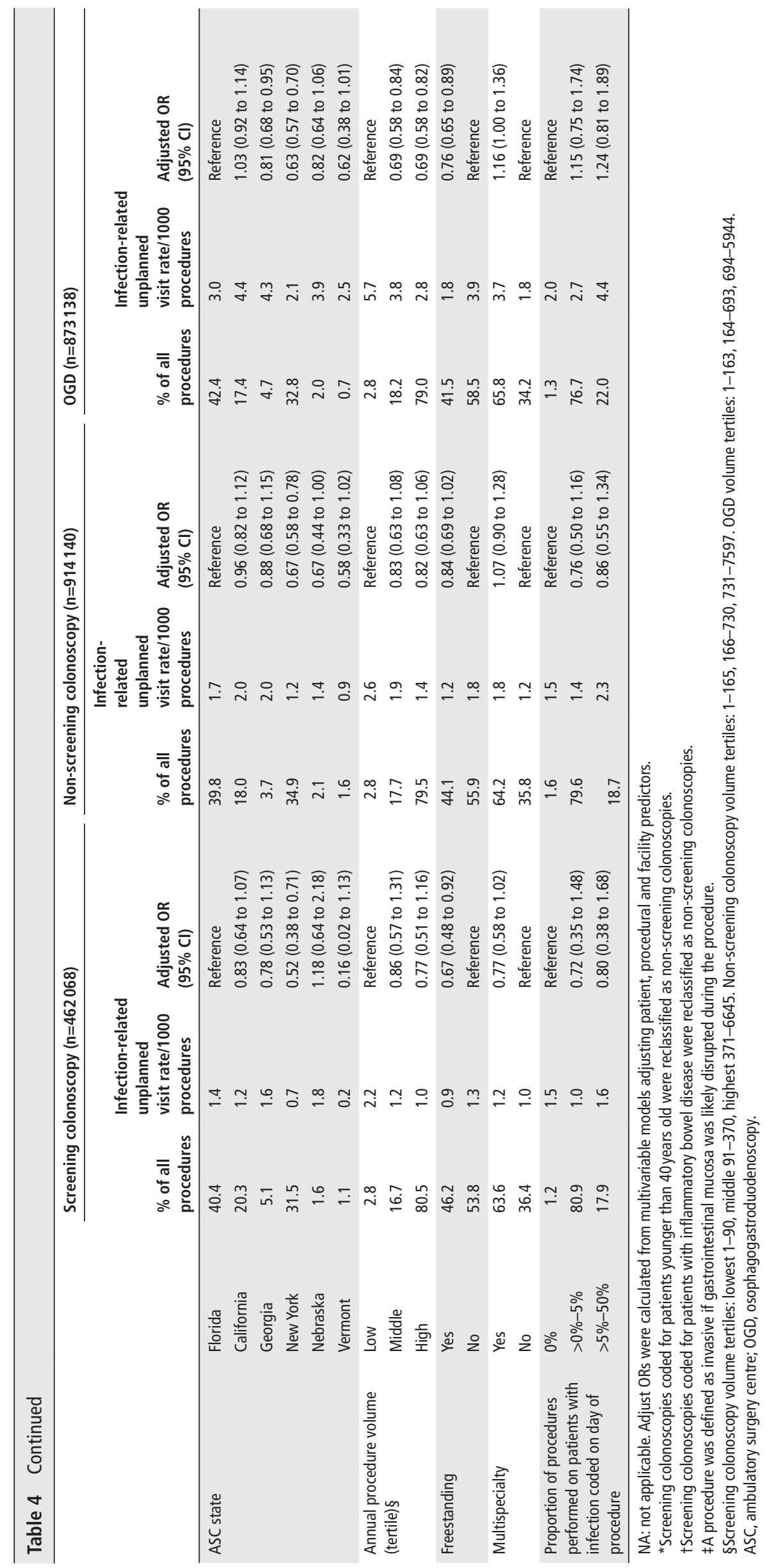




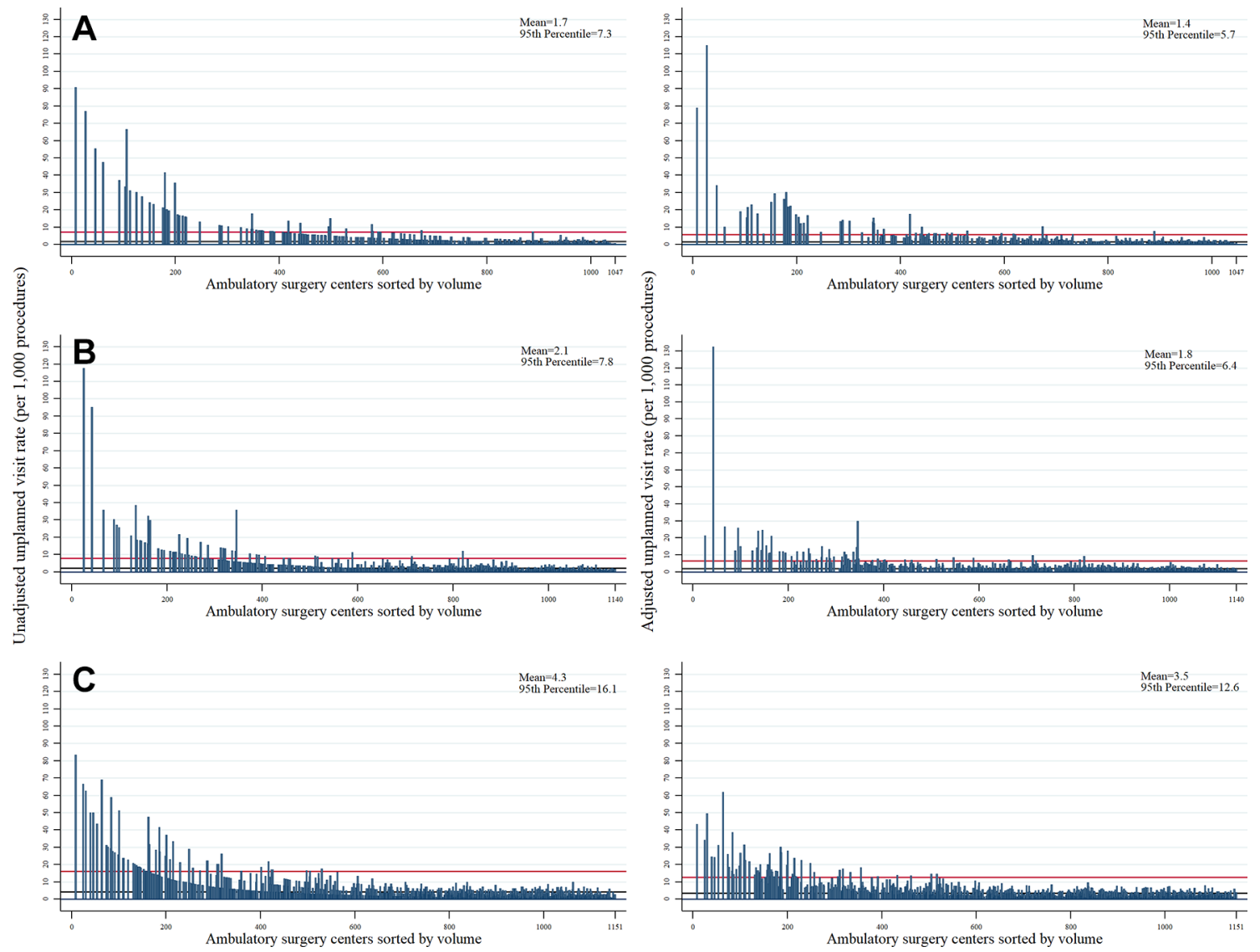

Figure 1 Volume-driven variation in the rates of infection-related unplanned visits within 7 days after colonoscopy or OGD among ASCs performing 10 or more procedures in 2014. (A) Screening colonoscopy (number of ASCs=1047). (B) Non-screening colonoscopy (number of ASCs=1140). (C) OGD (number of ASCs=1151). The graph on the left shows the unadjusted unplanned visit rates. The graph on the right shows the adjusted unplanned visit rates, adjusting for patient and procedure predictors listed in table 4. Each vertical navy bar in the graph represents an ASC. The horizontal black line indicates the mean rate. The horizontal red line indicates the 95th percentile. ASC, ambulatory surgery centre; OGD, osophagogastroduodenoscopy.

performed 10 or more OGDs ranged from 0 to 62 per 1000 procedures. In contrast, we observed small variation in the adjusted infection rates after screening mammography, ranging from 0 to 8 per 1000 examinations for the 220 ASCs with examination volume of 10 or greater.

\section{DISCUSSION}

Postendoscopic infections occur in more than 1 out of 1000 procedures for screening colonoscopy and more than 3 out of 1000 for OGD. These rates are twofold to fivefold higher than the infection rate after a screening mammography, but lower than bronchoscopy and cystoscopy. About 1 out of 5 postcolonoscopic infections and 1 out of 8 post-OGD infections involve the gastrointestinal system, 5-10 times higher than the rate of gastrointestinal infections after screening mammography. The rate of postendoscopic infections varies widely by ASC with a range of $0 \%-12.3 \%$ for screening colonoscopy, $0 \%-12.8 \%$ for non-screening colonoscopy and $0 \%-4.7 \%$ for OGD. ASC annual procedure volume is the strongest facility factor associated with risk of infection with the lowest relative risk after procedures performed in high-volume ASCs. Patients who were hospitalised within 30 days prior to a procedure have a greater than fivefold risk of postendoscopic infection compared with non-hospitalised patients. These findings have implications for the choice of facility to undergo an endoscopic procedure. The timing of procedures after hospitalisation should be carefully considered to prevent postendoscopic infections among recently hospitalised patients.

Despite the millions of endoscopic procedures performed annually in the USA, prior studies have not comprehensively examined postendoscopic infections. One study based on all-payer claims of California between 2005 and 2011 investigated the rate of pneumonia and bacteraemia after colonoscopy. ${ }^{20}$ They identified a 7-day pneumonia rate of 2.7-4.4 per 10000 procedures that was comparable with our estimate of 2.9-3.0 in 2014. Their 7-day bacteraemia (ICD-9-CM 790.7) rate estimate of $0.2-0.3$ per 10000 procedures was lower than our estimate of 1.9-2.6 for septicaemia (ICD-9-CM 038). We also calculated rates for a larger group of infections and comparison groups in our comprehensive examination.

Other studies mainly focused on cases of specific infections identified in outbreak investigations. Case reports published between 1981 and 1999 documented four cases of hepatitis $\mathrm{C}$ and two cases of hepatitis B after flexible sigmoidoscopy or colonoscopy and attributed to inadequate device cleaning. ${ }^{21-24}$ Between 1974 and 2001, 138 Pseudomonas aeruginosa infection cases and 3 hepatitis B cases were reported after OGD and also attributed to inadequate cleaning. ${ }^{25-32}$ According to a systematic review, there were only seven identified cases of endocarditis between 1966 and 2002 associated with OGD in the USA. ${ }^{33} 34$ However, we identified six endocarditis cases within 7 days after OGD in a single year in a $31 \%$ sample of the US population. 
Our findings suggest that postendoscopic infections are occurring without being detected by existing surveillance systems. Although the rates of infection after colonoscopy and OGD are higher than previously thought, they are still relatively low compared with other endoscopic procedures such as bronchoscopy and cystoscopy. The comorbidities and indications across the endoscopic procedures likely drive these rate differences. Prior encounters with the healthcare system were strong predictors of infections after gastrointestinal procedures.

Microorganisms thought to be most closely related to gastrointestinal endoscopic procedures include E. coli, Klebsiella spp., P. aeruginosa and Salmonella spp. ${ }^{34}$ We did observe relatively high rates of infections due to these organisms in the 2014 administrative claims data. For colonoscopy and OGD, the 7-day postendoscopic infection rate ranged from 0.084 to 0.199 per 1000 procedures for E. coli infection, 0.022-0.064 for K. pneumoniae and 0.008-0.047 for Pseudomonas, 3-fold to 24-fold higher compared with postmammography. This might also suggest that claims data have value for evaluating postendoscopic infection outcomes. While focusing on infections within 7 days maximised the specificity, infections with longer incubation periods were likely underestimated. For example, it was possible that we underestimated the rate of human papillomavirus infection. A lab-based study suggested human papillomavirus was not adequately killed by the disinfectants used for high-level disinfection. ${ }^{35}$

Hospitalisation for infectious pneumonia was common among the infections. We evaluated the infectious pneumonia codes (ICD-9-CM codes: 482.89, 482.9 and 483.8) that could represent misclassified aspiration pneumonia. ${ }^{16}$ Our results showed that these three codes only consisted a small fraction of all infectious pneumonia (2.8\%-3.7\%), suggesting that very few of the infectious pneumonia cases we identified were potentially misclassified aspiration pneumonia. In addition, the twofold higher rates of infectious pneumonia after colonoscopy or OGD compared with screening mammography suggests that endoscopy is associated with greater rates than the background occurrence of pneumonia. Our estimates of 7-day respiratory infection rates after screening mammography and prostate screening were 0.4 and 0.9 per 1000 , lower than the estimates reported for another background comparison group, healthcare workers. Two Chinese studies of 223 and 481 healthcare workers in emergency or respiratory wards reported the weekly incidence of clinical respiratory infection to be around 18/1000 for viral or bacterial infection. ${ }^{35} 36$

We found no significant difference in the risk of infections or aspiration pneumonia between colonoscopy procedures performed with and without general anaesthesia. Our estimates of aspiration pneumonia rate were 0, 0.119 and 0.341 per 1000 procedures for screening, non-screening colonoscopy and OGD. These estimates were comparable with a prospective multicentre study (ProSed2) that observed an aspiration event rate of 0.23 per 1000 gastrointestinal endoscopy procedures performed under sedation. ${ }^{37}$

Our findings are of particular importance for ASCs, which usually do not have their own infection control units and to which patients are unlikely to come back to seek care for their postendoscopic infections. These factors greatly impede the identification of infection-related complications at ASCs and can result in undetected infectious outbreaks that can profoundly harm many patients. Our study shows that ASCs had great variation in the rate of infection-related complications even after accounting for patient and procedure complexity. The infection rates observed at some low-procedure-volume ASCs were over 100 times more than the rates we would expect to see had patients received procedures at an average ASC. Therefore it is critically important for providers and patients to know the ASC-specific risks of postendoscopic infections for informed decision making about colorectal cancer screening and the most appropriate approach to diagnose and treat symptomatic conditions. Individuals with average risk of colorectal cancer should consider receiving non-invasive tests such as faecal occult blood test and faecal immunochemical test annually or biannually or a combination of colonoscopy and non-invasive tests at appropriate intervals as recommended by their national health policies. ${ }^{38} 39$ Similarly, judicious use of endoscopic procedures shortly after hospitalisation or increased vigilance or prophylaxis for infection should be considered as these patients had the highest risk of postendoscopic infections. Disposable endoscopes could be considered for patients at high risk of acquiring or transmitting infections. ${ }^{4041}$

Our all-cause unplanned visit findings were similar to the estimate of 16.3 all-cause unplanned visits within 7 days per 1000 colonoscopies in the $20 \%$ sample of 2010 Medicare data used to create the CMS measure. ${ }^{9}$ However, the CMS colonoscopy quality measure (ID 2086) has been questioned by experts in the gastroenterology community due to concerns that it might disincentivise endoscopic resection of precancerous lesions. Biopsies are associated with perforation and bleeding and may increase the all-cause unplanned visit rate, despite the benefit of biopsies for patients. Focusing on all-cause visits may also disincentivise providers to treat the most challenging patients at higher risk of a procedure-associated complication. ${ }^{36}$ In contrast, our facility-level adjusted infection-related unplanned visit rate specifically reflects the real quality of care, as the rate of postendoscopic infections should be zero, especially for screening colonoscopy.

The infection rates in this study can serve as a baseline estimate of the current burden of postendoscopic infection-related complications. Our findings can also serve as the scientific foundation for decision makers to identify if targeted interventions are needed in endoscopy units at ASCs to prevent the incidence of postendoscopic infections, similar to the interventions targeted to surgical and intensive care units to prevent the healthcare-associated infections in those settings.

Our study has a few limitations. We used all-payer claims data from six states in the USA because linkable all-payer data are not available nationally. Our results are likely generalisable to the whole country because together these six states have similar sex, racial and age composition as the national population and make up $31 \%$ of the US population. Although we examined the history of hospitalisation and endoscopic procedure within 30 days prior to the procedure as predictors and analysed unplanned visits within 7 days after the procedure as the outcome, we did not wash out procedures performed during the first 30 days and the last 7 days in 2014 due to lack of real service utilisation dates and months in the claims data of some states. This could result in an underestimation of the infection rates because we were not able to capture all possible outcomes for procedures performed during the last 7 days of 2014 and were not able to adjust for all hospitalisations that occurred 30 days prior. Our study shares the general limitations of claims-based research, such as flaws in billing codes and limited clinical details to determine causality. The procedure codes for sedation (CPT codes: 00810, 99143-99145, 99148-99150) was only coded in $1.0 \%$ of colonoscopy and $0.9 \%$ of OGD cases, so we were not able to study the impact of sedation on infection rates in most states, a limitation shared by another study using the same database. ${ }^{20}$ It is difficult to prove a definite causal relationship between the 
endoscopic procedures and infections without individual case investigation and microbiological testing of the endoscopes and facility environment. However, we tried to maximise the specificity of our outcome definition by excluding patients with a diagnosis of infection at the time of procedure and restricting the timeframe of infections to 7 days after procedures for the primary analyses. Because only 2014 data were available in this claims database, we were not able to compare the long-term risks and benefits between colonoscopy, OGD and non-invasive alternatives.

\section{CONCLUSION}

Our results suggest that infection-related complications after colonoscopy and OGD were much more common than previously thought and that the adjusted infection rates varied widely by ASC. Quality reporting may prevent ASC-specific postendoscopic infections. In addition, these data could inform shared decision making.

Contributors PW participated in study design, data analysis and wrote the first draft of the manuscript. SH conceived the study idea, oversaw the research project and participated in study design, data analysis and manuscript drafting and editing. SN and AK contributed to study design and manuscript editing. TX and MAM contributed to manuscript editing.

Funding This project was funded by the United States Department of Health and Human Services Agency for Healthcare Research and Quality (Grant number: 1R03HS024806-01).

Competing interests None declared.

Patient consent Not required.

Ethics approval Johns Hopkins Medicine Institutional Review Board.

Provenance and peer review Not commissioned; externally peer reviewed.

(c) Article author(s) (or their employer(s) unless otherwise stated in the text of the article) 2018. All rights reserved. No commercial use is permitted unless otherwise expressly granted.

\section{REFERENCES}

1 Design of Endoscopic Retrograde Cholangiopancreatography (ERCP). Duodenoscopes May Impede Effective Cleaning: FDA Safety Communication. 2015 https://www.fda. gov/medicaldevices/safety/alertsandnotices/ucm434871.htm (accessed 9 Jun 2017).

2 Los Angeles Times. Olympus' redesigned scope is linked to infection outbreak. 2017 http://www.latimes.com/business/la-fi-olympus-scope-outbreak-20170322-story.htm (accessed 9 June 2017).

3 Hutfless SM, Kalloo AN. Commentary on the 2016 multi-society task force endoscope reprocessing guidelines. Gastroenterology 2017;152:494-6.

4 Peery AF, Dellon ES, Lund J, et al. Burden of gastrointestinal disease in the United States: 2012 update. Gastroenterology 2012;143:1179-87.

5 Joseph DA, Meester RG, Zauber AG, et al. Colorectal cancer screening: estimated future colonoscopy need and current volume and capacity. Cancer 2016;122:2479-86.

6 Rathgaber SW, Wick TM. Colonoscopy completion and complication rates in a community gastroenterology practice. Gastrointest Endosc 2006;64:556-62.

7 Rabeneck L, Saskin R, Paszat LF. Onset and clinical course of bleeding and perforation after outpatient colonoscopy: a population-based study. Gastrointest Endosc 2011;73:520-3.

8 Ko CW, Riffle S, Michaels L, et al. Serious complications within 30 days of screening and surveillance colonoscopy are uncommon. Clin Gastroenterol Hepatol 2010;8:166-73

9 Ranasinghe I, Parzynski CS, Searfoss R, et al. Differences in colonoscopy quality among facilities: development of a post-colonoscopy risk-standardized rate of unplanned hospital visits. Gastroenterology 2016;150:103-13.

10 Reumkens A, Rondagh EJ, Bakker CM, et al. Post-colonoscopy complications: a systematic review, time trends, and meta-analysis of population-based studies. Am J Gastroenterol 2016;111:1092-101.

11 Voelker R. Ambulatory centers match hospital safety for outpatient colonoscopy, study says. JAMA 2011:305:2508-9.

12 ASCs. A positive trend in health care. 2011 http://www.ascassociation.org/advancin gsurgicalcare/aboutascs/industryoverview/apositivetrendinhealthcare (accessed 9 Jun 2017).
13 American Gastroenterological Association. Coding FAQ - screening colonoscopy. $2013 \mathrm{http}: / /$ www.gastro.org/practice-management/coding/screening-colonoscopy-faq (accessed 9 Jun 2017).

14 CMS.gov. Centers for medicare \& medicaid services measures inventory. 2017 https://www.cms.gov/Medicare/Quality-Initiatives-Patient-Assessment-Instruments/ QualityMeasures/CMS-Measures-Inventory.html (accessed 9 Jun 2017).

15 Corporation/Center for Outcomes Research and Evaluation (CORE). 2016 Measure Updates and Specifications Report Facility 7-Day Risk-Standardized Hospital Visit Rate after Outpatient Colonoscopy: A Quality Measure for Profiling Facility Performance Using Claims Data Version 2.0. 2016 https://www.cms.gov/Medicare/ Quality-Initiatives-Patient-Assessment-Instruments/HospitalQualitylnits/Downloads/ Colonoscopy_Update_Report_May2016_0QR.pdf.

16 Cooper GS, Kou TD, Rex DK. Complications following colonoscopy with anesthesia assistance: a population-based analysis. JAMA Intern Med 2013;173:551-6.

$17 \mathrm{Xu} \mathrm{B}$, Boero IJ, Hwang L, et al. Aspiration pneumonia after concurrent chemoradiotherapy for head and neck cancer. Cancer 2015;121:1303-11.

18 McCarthy EP, lezzoni LI, Davis RB, et al. Does clinical evidence support ICD-9-CM diagnosis coding of complications? Med Care 2000;38:868-76.

19 Elixhauser A, Steiner C, Harris DR, et al. Comorbidity measures for use with administrative data. Med Care 1998;36:8-27.

20 Wang L, Mannalithara A, Singh G, et al. Low rates of gastrointestinal and nongastrointestinal complications for screening or surveillance colonoscopies in a population-based study. Gastroenterology 2018;154:540-55.

21 Davis AR, Pink JM, Kowalik AM, et al. Multiple endoscopies in a Sydney blood dono found positive for hepatitis B and C antibodies. Med J Aust 1996;164:571.

22 Bronowicki JP, Venard V, Botté C, et al. Patient-to-patient transmission of hepatitis $C$ virus during colonoscopy. N Engl J Med 1997;337:237-40.

23 Le Pogam S, Gondeau A, Bacq Y. Nosocomial transmission of hepatitis C virus. Ann Intern Med 1999;131:794.

24 Federman DG, Kirsner RS. Leukocytoclastic vasculitis, hepatitis B, and the risk of endoscopy. Cutis 1999;63:86-7.

25 Morris IM, Cattle DS, Smits BJ. Letter: endoscopy and transmission of hepatitis B. Lancet 1975;2:1152.

26 Seefeld U, Bansky G, Jaeger M, et al. Prevention of hepatitis B virus transmission by the gastrointestinal fibrescope. Successful disinfection with an aldehyde liquid. Endoscopy 1981;13:238-9.

27 Birnie GG, Quigley EM, Clements GB, et al. Endoscopic transmission of hepatitis B virus. Gut 1983;24:171-4

28 Greene WH, Moody M, Hartley R, et al. Esophagoscopy as a source of pseudomonas aeruginosa sepsis in patients with acute leukemia: the need for sterilization of endoscopes. Gastroenterology 1974;67:912-9.

29 Brayko CM, Kozarek RA, Sanowski RA, et al. Bacteremia during esophageal variceal sclerotherapy: its cause and prevention. Gastrointest Endosc 1985;31:10-12.

30 Schoutens-Serruys E, Rost F, Depre G, et al. The significance of bacterial contamination of fiberoptic endoscopes. J Hosp Infect 1981;2:392-4.

31 Alvarado CJ, Stolz SM, Maki DG. Nosocomial infections from contaminated endoscopes: a flawed automated endoscope washer. An investigation using molecular epidemiology. Am J Med 1991;91:S272-80

32 Centers for Disease Control (CDC). Nosocomial infection and pseudoinfection from contaminated endoscopes and bronchoscopes--Wisconsin and Missouri. MMWR Morb Mortal Wkly Rep 1991;40:675-8.

33 Nelson DB. Infectious disease complications of GI endoscopy: Part I, endogenous infections. Gastrointest Endosc 2003:57:546-56.

34 Nelson DB. Infectious disease complications of GI endoscopy: part II, exogenous infections. Gastrointest Endosc 2003:57:695-711.

35 Maclntyre CR, Wang Q, Rahman B, et al. Efficacy of face masks and respirators in preventing upper respiratory tract bacterial colonization and co-infection in hospital healthcare workers. Prev Med 2014;62:1-7.

36 Raina MacIntyre C, Chughtai AA, Zhang Y, et al. Viral and bacterial upper respiratory tract infection in hospital health care workers over time and association with symptoms. BMC Infect Dis 2017;17:553.

37 Behrens A, Kreuzmayr A, Manner H, et al. Acute sedation-associated complications in Gl endoscopy (ProSed 2 Study): results from the prospective multicentre electronic registry of sedation-associated complications. Gut 2018:gutjnl-2015-311037.

38 Stracci F, Zorzi M, Grazzini G. Colorectal cancer screening: tests, strategies, and perspectives. Front Public Health 2014;2:210.

39 Matsuda T, Ono A, Kakugawa Y, et al. Impact of screening colonoscopy on outcomes in colorectal cancer. Jpn J Clin Oncol 2015;45:900-5.

40 Gluck N, Melhem A, Halpern Z, et al. A novel self-propelled disposable colonoscope is effective for colonoscopy in humans (with video). Gastrointest Endosc 2016;83:998-1004.

41 Huynh DK, Toscano L, Phan VA, et al. Ultrathin disposable gastroscope for screening and surveillance of gastroesophageal varices in patients with liver cirrhosis: a prospective comparative study. Gastrointest Endosc 2017;85:1212-7. 